\title{
A Fast Path Recovery Mechanism for MPLS Networks
}

\author{
Jenhui Chen ${ }^{1}$, Chung-Ching Chiou ${ }^{2}$, and Shih-Lin $\mathrm{Wu}^{1}$ \\ 1 Department of Computer Science and Information Engineering \\ Chang Gung University, Taoyuan, Taiwan 333, R.O.C. \\ \{jhchen, slwu\}@mail. cgu. edu.tw \\ 2 BroadWeb Inc., 24-1, Industry East Rd., IV, \\ Science Based Industrial Park, Hsin-Chu, Taiwan 300, R.O.C. \\ joe@broadweb.com.tw
}

\begin{abstract}
The major concept of the Multi-Protocol Label Switching (MPLS) network uses the Label Switch Path (LSP) technique that provides high performance in packet delivery without routing table lookup. Nevertheless, it needs more overhead to rebuild a new path when occurring link failure in the MPLS network. In this paper, we propose an efficient fast path recovery mechanism, which employs the Diffusing Update Algorithm (DUAL) to establish the working and backup paths concurrently and modify the Label Distribution Protocol (LDP) to establish the LSP by using Enhanced Interior Gateway Routing Protocol (EIGRP). Simulation results show that the proposed mechanism not only improves resource utilization but provides shorter path recovery time than the end-to-end recovery mechanism.
\end{abstract}

\section{Introduction}

In connectionless network protocols, an independent forwarding decision is made in each switch router when a packet is delivered from one router to next router. Traditionally, in the IP network, each router runs a network layer routing algorithm (e.g., Dijkstra algorithm) for supporting route setup procedure. Current routing algorithm, despite being robust and survivable, can take a substantial amount of time to recovery when a failure occurs, which can be on the order from several seconds to minutes and can cause serious disruption of service in the interim. This is unacceptable for many applications that require highly reliable service. Thus, the Internet service provider may need an efficient path protection mechanism to minimize the recovery time when link failure, and maximize the network reliability and survivability.

Path-oriented technologies such as Multi-Protocol Label Switching (MPLS), which is described in the RFC3031 of Internet Engineering Task Force (IETF) [4, 8], can be used to enhance the reliability of IP networks. A fundamental concept of MPLS networks, which consists of Label Edge Routers (LERs) around a core of meshed Label Switching Routers (LSRs), is to use small labels for routing. In order to carry the same labeled traffic in MPLS networks, a label assignment 
distribution scheme, Label Distribution Protocol (LDP) [1], is taken to establish the Label Switched Path (LSP) beforehand.

To protect an LSP in MPLS networks, a protection LSP is proposed to establish the working and backup paths at the same time in the initial setup process. When a network failure is detected, the MPLS will perform a path recovery mechanism by only switching working traffic onto the backup path. However, this recovery mechanism does not switch the working path to backup path efficiently and leads the degradation of network performance due to its long processing delay. Therefore, in this paper, we propose a fast path recovery mechanism, which employs the Enhanced Interior Gateway Routing Protocol (EIGRP) and the Diffusing Update Algorithm (DUAL) [5] together, to find the working and backup paths simultaneously and modify the LDP to establish the LSP by using the routing table of EIGRP. Moreover, the proposed path recovery mechanism would not occupy any available bandwidth in MPLS networks.

The remainder of this paper is organized as follows. Section 2 describes the proposed recovery mechanism in details. The simulation models and results are shown in Section 3. Finally, we give some conclusions in Section 4.

\section{The Fast Path Recovery Mechanism}

\subsection{An Overview}

The IETF has proposed two types of recovery models for the MPLS-based LSP: the protected switching model and the rerouting model $[2,6,10]$. However, there are two critical drawbacks that can be improved. One drawback is that the setup process needs at least two round-trip times to establish the working and backup paths. Another drawback is that the backup path cannot be found from the routing table due to its limited information. To overcome these drawbacks, we use the DUAL to converge after an arbitrary sequence of link cost or topological changes in a finite time and guarantee a loop-free routing. And employ the EIGRP, which is the Cisco proprietary protocol and a modified protocol stack of the MPLS, to establish working and backup paths by using the information of the successor and feasible successor indicated in EIGRP routing table.

\subsection{The Recovery Mechanism}

In the proposed LDP, we added additional parameters to the optional parameters field each of the label request message (LRM) and the label mapping message (LMM), which is shown in Fig. 1. The LRM contains a successor LRM (SLRM) and a feasible successor LRM (FSLRM), respectively. The SLRM is used to request the label of working path according to the successor of routing table and FSLRM is used to request label of backup path according to the feasible successor of routing table. The LMM contains the successor LMM and the feasible successor LMM, which are used to map label of working path and backup path, respectively. In addition, shown in Table 1, the Label Information Base (LIB) 


\begin{tabular}{|c|c|c|}
\hline 0 & Label Request & Message Length \\
\hline \multicolumn{2}{|c|}{ Message ID } \\
\hline FEC TLV \\
\hline Optional Parameters
\end{tabular}

(a) Label request message of LDP

1 2

01234567890123456789012345678901

\begin{tabular}{|c|c|c|}
\hline 0 & Label Mapping & Message Length \\
\hline \multicolumn{2}{|c|}{ Message ID } \\
\hline FEC TLV \\
\hline Label TLV \\
\hline
\end{tabular}

(b) Label mapping message of LDP

Fig. 1. The packet format of LRM and LMM.

Table 1. An Example of modified LIB

\begin{tabular}{|c|c|c|c|c|c|}
\hline \multicolumn{5}{|c|}{ LER S } \\
\hline In/I & In/L & FEC & Out/I & Out/L & State \\
\hline 0 & 25 & \multirow{2}{*}{163.25 .101} & 1 & 21 & active \\
\cline { 3 - 6 } & & 2 & 20 & backup \\
\hline
\end{tabular}

is modified to add an additional state field to record which label is assigned to working path or backup path.

Fig. 2 shows the proposed LDP algorithm in details. An example of using the proposed algorithm is given in Fig. 3. The Fig. 3(b) shows our recovery mechanism is belong to the protection switch model with partial backup. When some links of the working path experience failure, it can switch over the backup path rapidly since it does not need to send a notification to the source node.

\section{Simulation Results}

In this section, we evaluated the performance of proposed mechanism by carrying out simulation studies on regular network topologies in different mesh sizes: $5 \times 10$, $5 \times 15,5 \times 20,5 \times 25,5 \times 30,5 \times 35$ and 5x40, respectively. We also implemented the end-to-end backup scheme to compare its performance in terms of average path restoration time, throughput, average LSP establishing time, average resource consumption, and average hop counts of backup path.

The network topology is a partial mesh topology as shown in Fig. 4. The bandwidth of each link is set as 10 units and the link delay time is dependent on link cost. Thus, the link delay along any link is proportional to its link cost. The traffic arrival rate is constant and maximum transmitting unit is fixed with 1,500 bytes. 


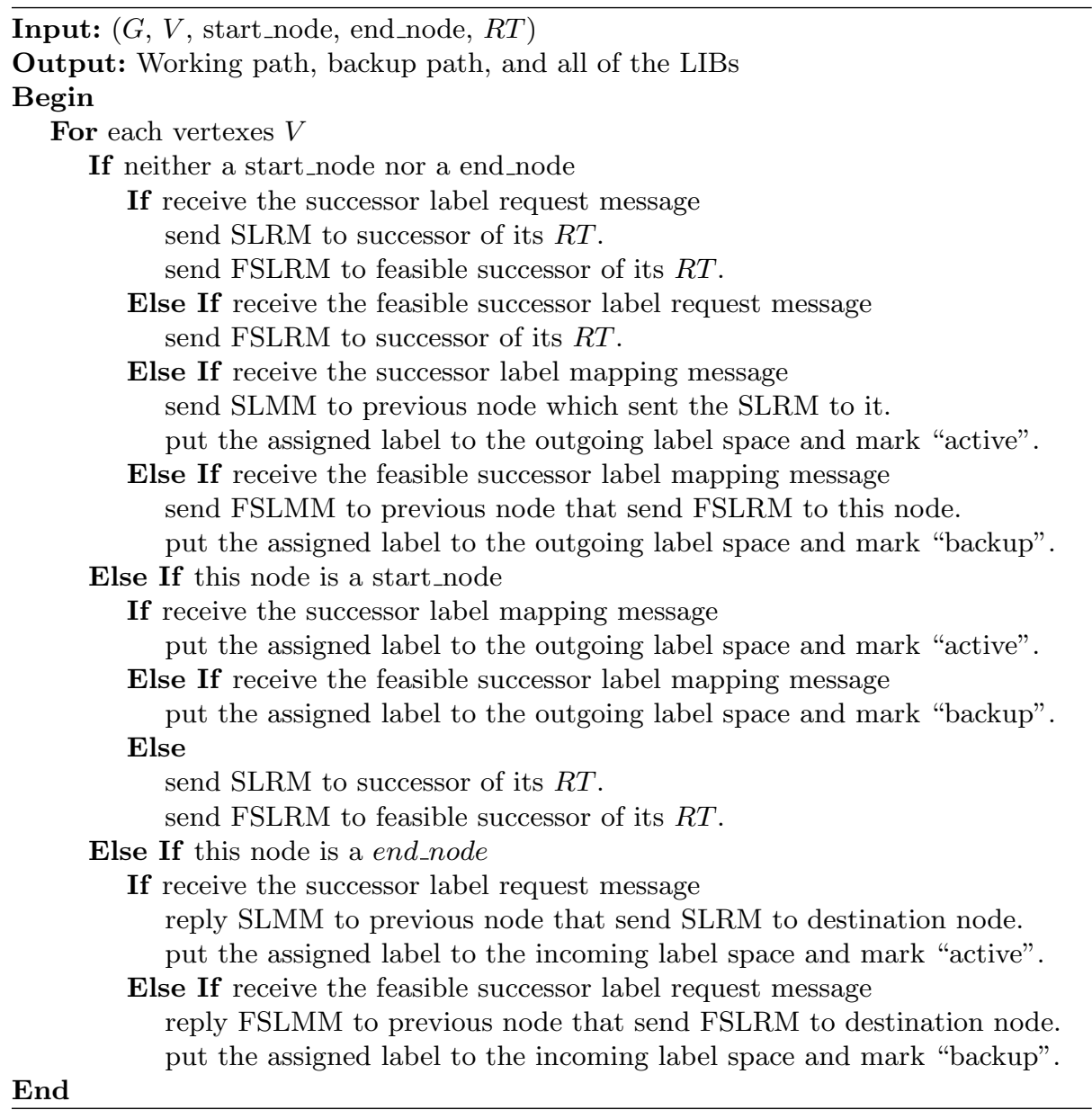

Fig. 2. The algorithm of modified LDP

In Fig. 5, we compare the packet delivery ratio of our scheme with the endto-end backup scheme's by varying the mesh size. We can see that both packet delivery ratios of proposed scheme and end-to-end scheme degrades when the the size of mesh increases. This is because that the end-to-end scheme performs the path recovery mechanism after a link failure is detected. On the contrary, in our scheme, a backup path is established with the working path simultaneously and, therefore, the path recovery time will be minimized. As a result, many packets will be queued in buffer and wait for another available route to the destination. This drawback will degrade the performance of MPLS networks.

Fig. 6 shows the comparison of average restoration (path recovery) time of two schemes. The path restoration time of the end-to-end backup scheme is getting longer with the length of working path due to the end-to-end rerouting 


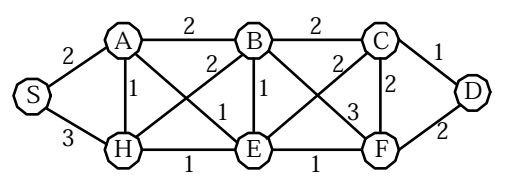

(a) The initial network topology

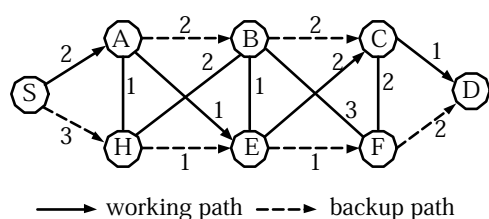

(b) The established LSP

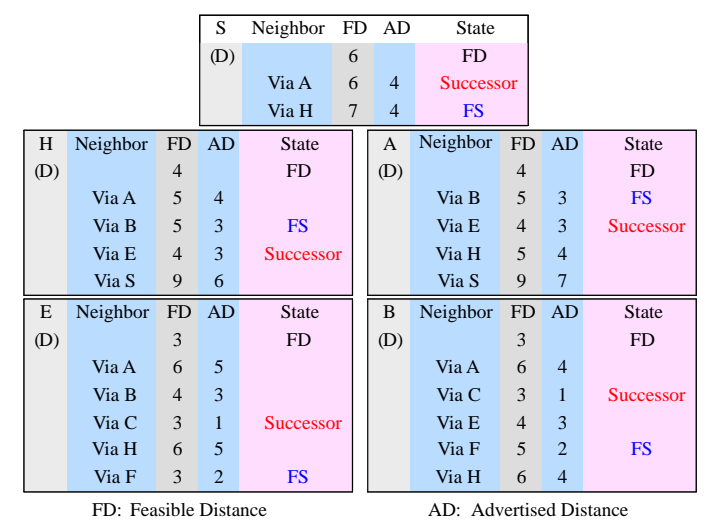

(c) The convergent routing table of the EIGRP

Fig. 3. An example of the LSP establishment by proposed LDP.

mechanism. However, the path restoration time of our scheme is a placid curve and keeps around $3 \mu$ s since it has a pre-established backup path.

In addition, the establish time of LSP in our proposed scheme is half of the end-to-end backup scheme since our scheme establishes working path and backup path simultaneously as shown in Fig. 7. Moreover, the end-to-end backup scheme must use two round-trip times to establish working path and backup path.

We observe the number of packet loss in end-to-end scheme and proposed scheme by varying the working path lengths. Fig. 8 shows the derived packet loss by sending 500 packets to a fixed destination. The average packet loss of proposed scheme does not exceed 10 packets since the backup path is pre-established. On the contrary, the number of packet loss of end-to-end scheme increases proportionally with the working path length due to a long delay of path recovery.

The curve of proposed scheme is going to up and down since current situation of EIGRP routing table might have the feasible successor or not. Thus, the resource consumption and backup path hop counts are depending on the number of feasible successors of the node in working path as shown in Fig. 9 and Fig. 10.

\section{Conclusion}

In this paper, a fast path recovery mechanism for LDP in MPLS networks is presented and investigated. The proposed mechanism using routing table of EIGRP 


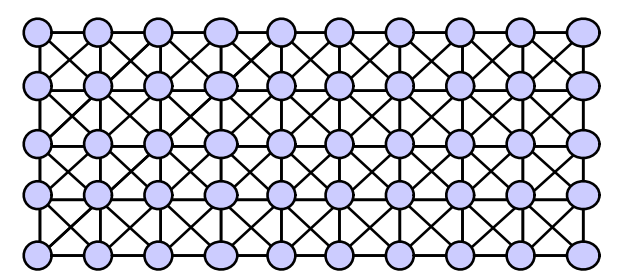

Fig. 4. An Example of mesh topology size with 5x10.

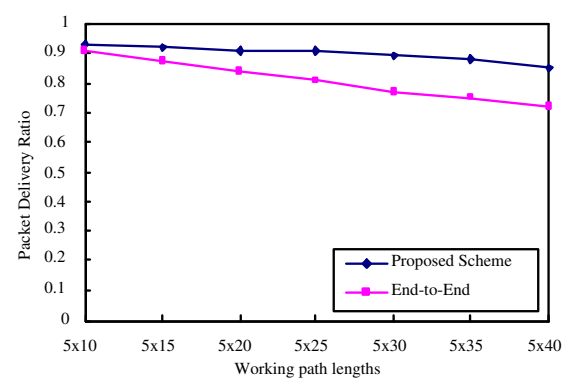

Fig. 5. The comparison of packet delivery ratio of proposed scheme and end-to-end backup scheme by varying the mesh size.

base on Diffusing Update Algorithm (DUAL) can converge rapidly of finding the working and backup paths. This mechanism not only improves resource utilization but provides faster failure recovery time than the end-to-end recovery mechanism. Simulation results show that the proposed mechanism can fast migrate ongoing data streaming to pre-established backup path efficiently without wasting bandwidth. This mechanism enable MPLS networks to provide high quality-of-service (QoS) applications sufficiently.

\section{Acknowledgment}

This work was supported by National Science Council, Taiwan, R.O.C., under Contract NSC93-2213-E-182-022.

\section{References}

1. L. Andersson, P. Doolan, N. Feldman, A. Fredette, and B. Thomas, "LDP Specification," IETF RFC 3036, Jan. 2001.

2. D. Awduche, J. Malcolm, J. Agogbua, M. O'Dell, and J. McManus, "Requirements for Traffic Engineering over MPLS," IETF RFC 2702, Sept. 1999.

3. A. Farrel, "Fault Tolerance for LDP and CR-LDP," IETF draft draft-ietf-mplsldp-ft-01.txt, Feb. 2001. 


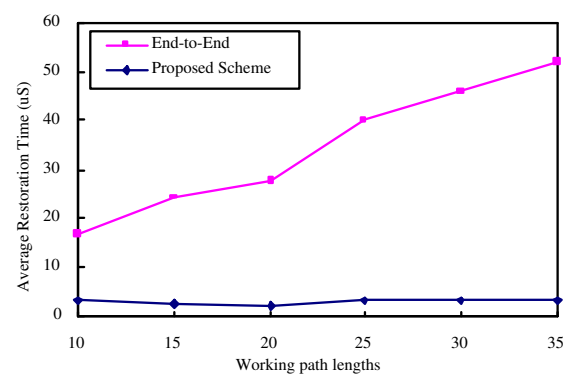

Fig. 6. The comparison of average restoration time of proposed scheme and end-to-end backup scheme by varying working path length.

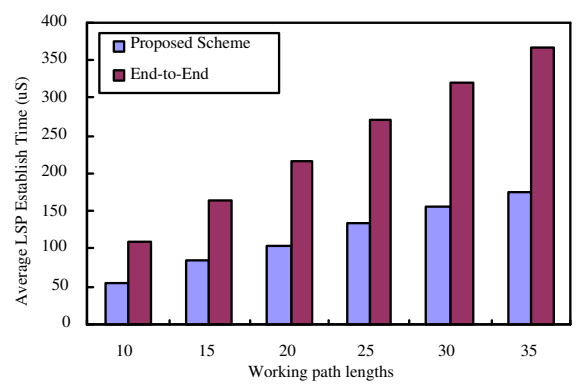

Fig. 7. The comparison of average LSP establish time of proposed scheme and end-toend backup scheme by varying working path length.

4. Future Software, "Multi Protocol Label Switching," white paper of Future Software Limited, India, 2002. http://www.futsoft.com.

5. J.J. Garcia-Lunes-Aceves, "Loop-free Routing Using Diffusing Computations," IEEE/ACM Trans. Networking, vol. 1, no.1, pp. 130-141, Feb. 1993.

6. C.C. Huang, V. Sharma, K. Owens, and S. Makam, "Building Reliable MPLS Networks Using a Path Protection Mechanism," IEEE Commun. Mag., vol. 40, no. 3, pp. 156-162, Mar. 2002.

7. J. Lawrence, "Designing Multiprotocol Label Switching Networks," IEEE Commun. Mag., vol. 39, no. 7, pp. 134-142, July 2001.

8. E. Rosen, A. Viswanathan, and R. Callon, "Multiprotocol Label Switching Architecture," IETF RFC 3031, 2001.

9. J. Wu, D. Y. Montuno, H.T. Mouftah, G. Wang, and A.C. Dasylva, "Improving the Reliability of the Label Distribution Protocol," in Proc. Local Comp. Networks, pp. 236-242, 2001.

10. S. Yoon, H. Lee, D. Choi, Y. Kim, G. Lee, and M. Lee, "An Efficient Recovery Mechanism for MPLS-based Protection LSP," in Proc. Int. Conf. ATM (ICATM'2001) and High Speed Intelligent Internet Symp., pp. 75-79, 2001. 


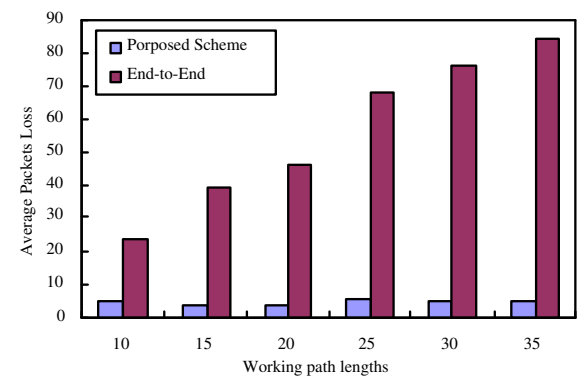

Fig. 8. The comparison of average packet loss of proposed scheme and end-to-end backup scheme by varying working path length.

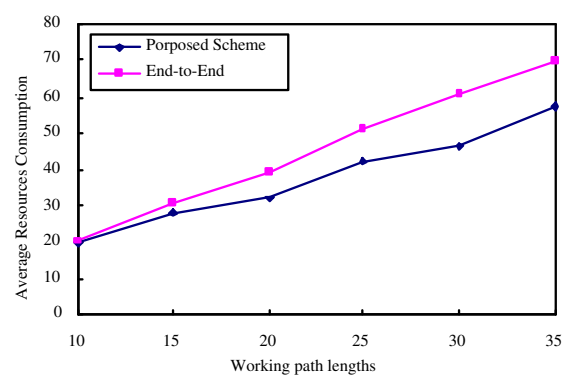

Fig. 9. The comparison of average resources consumption of proposed scheme and end-to-end backup scheme by varying working path length.

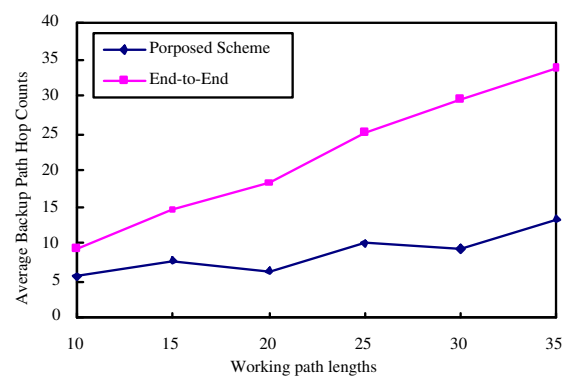

Fig. 10. The comparison of average hop counts of backup path of proposed scheme and end-to-end backup scheme by varying working path length. 